> Comment se développent les hépatocytes et les cholangiocytes dans le foie embryonnaire et adulte? Quelle est l'origine des cellules stellaires et sinusoïdales du foie? Un modèle, basé principalement sur des méthodes de traçage de lignées, émerge aujourd'hui et propose une filiation des cellules du foie embryonnaire au stade adulte, ainsi que dans un foie adulte en régénération. Ce modèle définit les potentiels de différenciation des hépatoblastes, des cellules de la plaque ductale, des cellules progénitrices du foie adulte, ainsi que du mésoderme embryonnaire. <

\section{Le développement des lignages hépatiques dans le foie normal et durant la régénération}

Frédéric Lemaigre

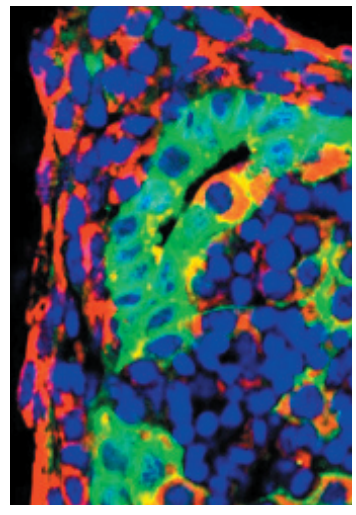

Institut de Duve, Université catholique de Louvain, avenue Hippocrate 75/B1-7503, 1200 Bruxelles, Belgique.

frederic.lemaigre@uclouvain.be
Les hépatocytes constituent la population de cellules épithéliales la plus importante du foie. Ils s'organisent en travées irradiant autour des espaces portes, et sont au contact les uns des autres par leurs pôles latéraux. Les hépatocytes exercent de multiples fonctions, dont la production de bile qui est sécrétée au pôle apical des cellules. Les pôles apicaux d'hépatocytes voisins se juxtaposent, délimitant ainsi d'étroits canalicules drainant la bile vers des canaux biliaires bordés de cholangiocytes. La jonction entre les canaux biliaires et les canalicules est assurée au niveau des passages de Hering dont la lumière est bordée d'hépatocytes et de cholangiocytes. Enfin, le foie est vascularisé par les flux sanguins de la veine porte et de l'artère hépatique qui convergent vers les sinusoïdes, ces derniers échangeant les métabolites avec les hépatocytes via l'espace de Disse, un espace fin où se logent les cellules stellaires (cellules de Ito) (Figure 1).

Cette organisation complexe se met progressivement en place durant la vie fœtale, ainsi que lors de la régénération hépatique chez l'adulte. Récemment, la question de l'origine des divers types de cellules hépatiques, chez le fœtus et l'adulte, a fait l'objet d'une attention particulière, notamment grâce à la mise en œuvre des techniques de traçage de lignées.

Vignette (photo @ Frédéric Lemaigre)

\section{Hépatocytes et cholangiocytes dérivent de précurseurs bipotents}

Les études embryologiques ont montré que le foie se développe à partir de l'endoderme sous l'influence de signaux provenant des tissus voisins [1, 2]. Ce développement, qui débute chez l'homme au $18^{\mathrm{e}}$ jour de grossesse et chez la souris au $9^{e}$ jour de gestation, se caractérise à ces stades par la formation d'un bourgeon cellulaire constitué d'une population en apparence homogène d'hépatoblastes. Combinant l'analyse de l'expression de gènes durant le développement du foie à une étude du potentiel de différenciation d'hépatoblastes en culture, Germain et al. [3] posaient en 1988 les bases d'un modèle prédisant que les hépatoblastes sont des cellules bipotentes, à l'origine des hépatocytes et des cholangiocytes intrahépatiques.

Ce modèle n'a pas été démenti par la suite. Bien qu'un traçage de lignée rigoureux n'ait pas été effectué, l'accumulation de données relatives à l'expression de gènes au cours du développement renforce la notion du caractère bipotent des hépatoblastes. À titre d'exemple, les hépatoblastes expriment les cytokératines (CK) 8, 18 et 19. À des stades plus tardifs, les cholangiocytes maintiennent l'expression des trois gènes, les hépatocytes perdant par contre graduellement celle de CK19 pour ne conserver que l'expression de CK8 et CK18 [4]. D'autre part, la détection en conditions pathologiques d'anomalies de ségrégation des lignages hépatocytaires et biliaires témoigne indirectement de l'origine commune des deux lignages, et donc du caractère bipotent des hépatoblastes en situation normale. En effet, chez certaines souris, notamment celles qui ne produisent plus les 


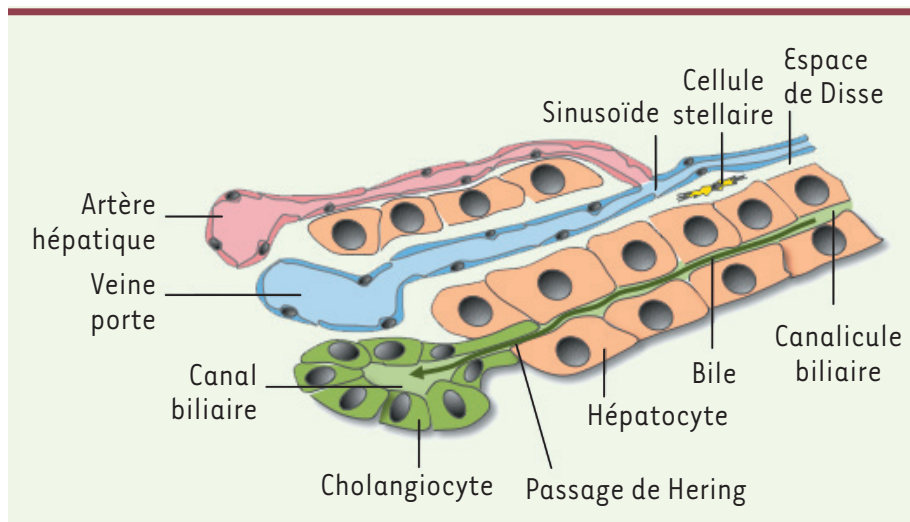

Figure 1. Organisation des cellules dans le foie adulte.

facteurs de transcription hepatocyte nuclear factor-6 (HNF6), onecut-2 (OC2) ou hematopoietically expressed homeobox factor (Hex), les cellules hépatiques présentent un phénotype mixte hépatobiliaire [5-7]. Celui-ci est caractérisé par la coexpression de protéines hépatocytaires et cholangiocytaires au sein des mêmes cellules, témoignant d'une anomalie de la ségrégation des lignages hépatocytaire et cholangiocytaire à partir des hépatoblastes. Une même anomalie de ségrégation de lignages hépatiques, caractérisée par l'apparition de cellules mixtes hépatobiliaires, est également observée chez le poisson zèbre (Danio rerio), suite à l'inhibition de l'expression de composants de la voie Notch [8]. Cette observation suggère que la détermination des lignages hépatiques s'opère de manière similaire entre les espèces. Enfin, notons que des cellules hépatobiliaires ont également été détectées chez l'homme dans le contexte du syndrome de Meckel, un syndrome polymalformatif associé à des anomalies de la morphogenèse biliaire et à un défaut de différenciation des hépatocytes et des cholangiocytes. Le foie des fœtus atteints de ce syndrome montre des canaux biliaires délimités irrégulièrement de cholangiocytes exprimant des protéines d'hépatocytes. En outre, les hépatocytes de ces fœtus présentent un phénotype mixte caractérisé par l'expression du marqueur biliaire CK19 [9].

\section{Morphogenèse des canaux biliaires}

Aux alentours de la $8^{e}$ semaine de grossesse chez l'homme, ou du $14^{e}$ jour de gestation chez la souris, les hépatoblastes donnent naissance aux précurseurs des cholangiocytes intrahépatiques. Cette différenciation s'opère à proximité des branches de la veine porte. Elle se caractérise par un renforcement de l'expression des cytokératines 8 , 18 et 19, et par l'apparition de protéines spécifiques aux cholangiocytes telles que Sox9 et l'ostéopontine [10]. Les précurseurs de cholangiocytes s'alignent autour du mésenchyme périportal pour constituer un manchon de cellules communément appelé plaque ductale [11]. La plaque ductale est composée, au départ, d'une monocouche de cellules à partir desquelles se forment les canaux biliaires. Par la suite, à certains endroits de la plaque ductale, des cellules dont le phénotype rappelle celui des hépatoblastes s'apposent sur la plaque ductale tout en créant une lumière, constituant ainsi une structure canalaire primitive. Ces canaux biliaires primitifs sont initialement asymétriques, étant délimités du côté de la veine porte par des cellules de plaque ductale, et du côté parenchymateux par des cellules au profil d'hépatoblaste (Figure 2). Progressivement, les canaux acquièrent une symétrie radiaire, toutes les cellules bordant les canaux présentant in fine les caractéristiques de cholangiocytes $[10,12]$.

\section{Destinée des cellules de la plaque ductale}

Seule une partie de la plaque ductale contribue à la formation des canaux biliaires. En moyenne, deux canaux sont formés à proximité de chaque branche de la veine porte, alors que la plaque ductale forme un manchon quasi continu autour du mésenchyme périportal [13]. Dès lors, qu'advient-il des cellules de la plaque ductale qui ne sont pas impliquées dans la morphogenèse des canaux biliaires? L'apoptose fut considérée pendant longtemps comme le mécanisme responsable de la disparition des cellules non impliquées dans la tubulogenèse [14]. Toutefois, à l'aide de marqueurs spécifiques de la plaque ductale et de l'apoptose, il s'est avéré impossible de soutenir cette hypothèse, tant chez l'homme que chez la souris [15]. Des expériences de traçage de lignées se sont alors imposées comme une solution permettant de déterminer la destinée de l'ensemble des cellules de la plaque ductale.

Dans une expérience de traçage de lignée, un type cellulaire précis est marqué génétiquement à un moment donné de la différenciation, et les descendants de ce type cellulaire sont suivis par détection de la marque génétique. En pratique, ce marquage est obtenu par recombinaison irréversible d'une séquence génique sous l'influence d'une recombinase Cre. Cette dernière n'est exprimée que dans le type cellulaire considéré et n'est active transitoirement qu'après administration de tamoxifène. Cette approche a été mise en oeuvre pour identifier la destinée des cellules de la plaque ductale [15]. Ces cellules exprimant Sox9, un transgène constitué des séquences codant la $\mathrm{Cre}$ inductible (creER, ER pour estrogen receptor) sous le contrôle des séquences régulatrices du gène Sox9 a été intégré dans le génome de souris [16]. L'expression de Sox9 en condition homéostatique est restreinte aux cellules de la plaque ductale chez l'embryon, et aux cholangiocytes chez l'adulte. II en résulte que la recombinase CreER est exprimée dans les mêmes cellules. Les souris Sox9-CreER ont été croisées avec d'autres possédant un locus ROSA26 n'exprimant la yellow fluorescent protein (YFP) qu'après élimination d'une cassette stop 


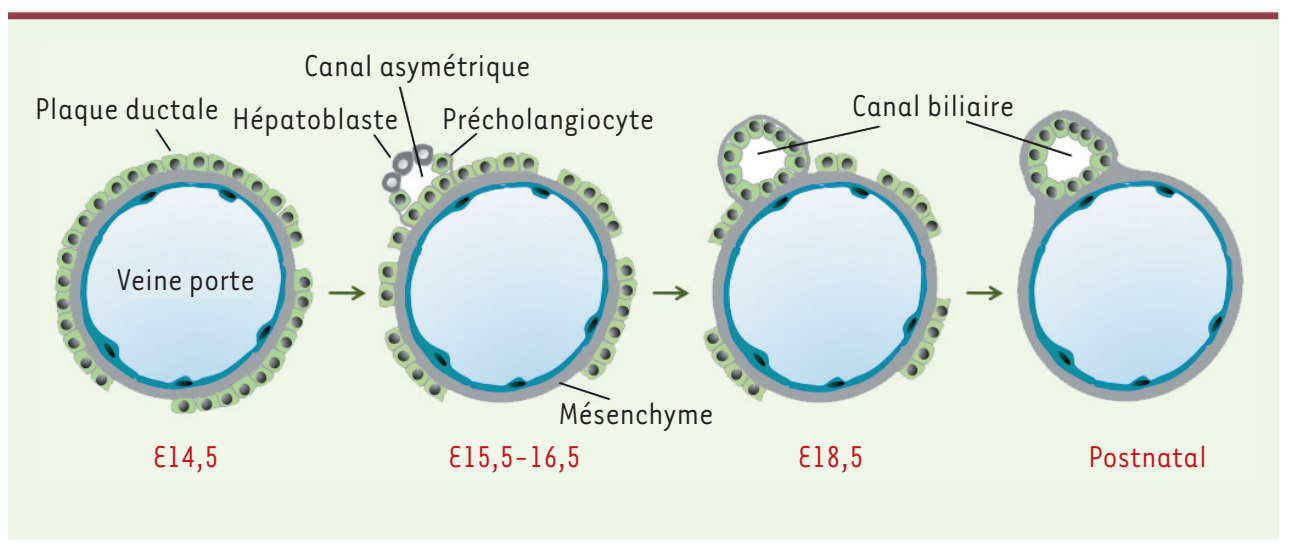

Figure 2. Morphogenèse des canaux biliaires. Les canaux biliaires se développent à partir de la plaque ductale durant un processus impliquant une phase transitoire d'asymétrie. Le décours temporel est mentionné pour la souris. $\varepsilon$, jour embryonnaire.

flanquée de sites loxP (Figure 3). Le tamoxifène a ensuite été administré aux femelles porteuses d'embryons Sox9-CreER; ROSA26R-YFP, à un stade où la plaque ductale était présente chez les fœtus (jour embryonnaire 15). Peu après la naissance, les souriceaux ont été collectés et l'expression de la YFP déterminée par immunomarquage sur coupes. Les résultats révélèrent que les cholangiocytes bordant les canaux biliaires exprimaient la YFP et dérivaient donc de la plaque ductale, comme prédit par les études histologiques. Toutefois, un certain nombre d'hépatocytes périportaux exprimaient également la YFP, démontrant que ceux-ci étaient issus de la plaque ductale, probablement à partir des cellules non impliquées dans la morphogenèse des canaux. En conséquence, le modèle de détermination des lignages hépatocytaire et cholangiocytaire prévoit maintenant que les hépatoblastes génèrent, d'une part, des précurseurs d'hépatocytes qui se développent en hépatocytes et, d'autre part, des précurseurs de cholangiocytes qui constituent une plaque ductale donnant naissance aux canaux biliaires ainsi qu'à certains hépatocytes périportaux (Figures 3 et 4).

\section{Origine mésodermique des cellules stellaires et sinusoïdales}

Les cellules stellaires (ou cellules étoilées) stockent de la vitamine A, et leur morphologie est caractérisée par de fins prolongements dendritiques. Elles s'activent à la suite d'une lésion hépatique, perdant leurs vésicules contenant la vitamine A. Elles acquièrent alors un phénotype myofibroblastique et produisent des cytokines inflammatoires et de la matrice extracellulaire, contribuant ainsi au développement d'une cirrhose. Les cellules stellaires présentent un phénotype mixte mésenchymateux et neuroendocrine. Elles expriment des protéines typiques du mésenchyme (desmine, vimentine) et de tissus neuroendocrines (N-CAM [neural cell adhesion molecule], neurotrophines), suscitant l'hypothèse que les cellules stellaires pourraient provenir soit du mésoderme, soit des crêtes neurales (neurectoderme). Des traçages de lignées en tous points comparables à ceux décrits plus haut, mais faisant appel à une Cre recombinase exprimée dans le mésoderme sous le contrôle $\mathrm{du}$ gène mesoderm posterior 1 (MesPl), ont révélé que les cellules stellaires présentent une origine mésodermique [17] (Figure 4). Le mésoderme donne par ailleurs naissance au septum transverse, le tissu mésenchymateux adjacent à l'endoderme et envahi par les hépatoblastes au stade initial du développement du foie. Un autre traçage de lignée, faisant appel à une Cre recombinase exprimée dans le septum transverse (WT1-CreER), a révélé que ce tissu produit les cellules mésothéliales et submésothéliales qui enveloppent le foie, et que certaines de ces cellules pénètrent dans le parenchyme hépatique pour y former des cellules stellaires [18]. L'hypothèse d'une origine neurectodermique des cellules stellaires fut éliminée, à nouveau au moyen d'une expérience de traçage de lignée : les souris Wntl-Cre; ROSA26R-YFP expriment la Cre recombinase et activent l'expression de YFP dans les crêtes neurales. Dans ce dernier modèle, aucune cellule stellaire n'exprime la YFP, éliminant donc les crêtes neurales comme source potentielle de ces cellules [19].

L'origine des cellules qui bordent les sinusoïdes a été étudiée dans l'embryon de poulet, au moyen d'une technique de traçage différente. Le foie est délimité par le mésothélium, et ce dernier s'avère capable de capter un traceur fluorescent en moins de $3 \mathrm{~h}$ après injection dans l'embryon. Quarante huit à 96 h après l'injection, on trouve le traceur dans les cellules sinusoïdales, suggérant que ces dernières sont issues du mésothélium [20]. Chez le poulet, le septum transverse se développe plus tardivement que le mésothélium, alors que chez la souris le mésothélium dérive du septum transverse. Ces particularités d'espèces mises à part, il est aujourd'hui très probable que le mésoderme donne naissance au septum transverse et au mésothélium, ces tissus constituant une source de cellules stellaires et sinusoïdales (Figure 4).

\section{Homéostasie et régénération cellulaire dans le foie adulte}

Le modèle de la détermination des lignages hépatiques chez l'embryon est actuellement peu controversé. II en est tout autrement dans le foie adulte. En conditions 


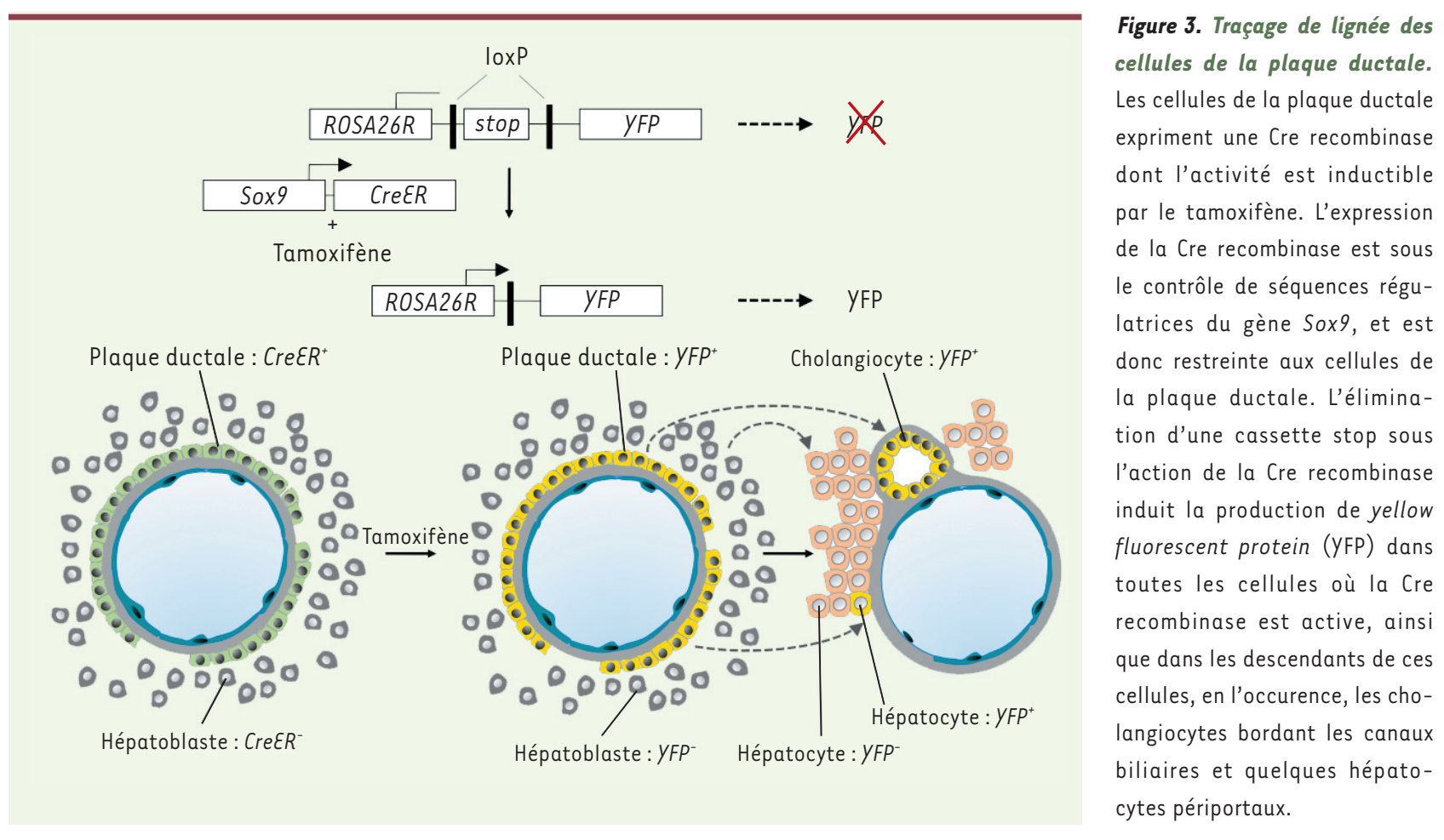

normales, le foie est considéré comme quiescent avec un taux de renouvellement cellulaire très faible. Toutefois, l'équipe de Uemoto suggère, sur la base d'expériences de traçage de lignée, que les cholangiocytes se comportent comme des précurseurs qui s'autorenouvellent, tout en alimentant le foie de manière continue en hépatocytes [21]. Ce modèle n'est cependant pas accepté par d'autres chercheurs. Notre équipe a montré que les cholangiocytes adultes prolifèrent très peu et ne génèrent pas de nouveaux hépatocytes [15]. En outre, au contraire de la période prénatale, l'administration de tamoxifène en période postnatale peut induire une expression ectopique de Sox9 dans certains hépatocytes. Sachant que l'équipe de Uemoto fait appel à des souris Sox9-CreeR qui sont censées n'exprimer la Cre recombinase que dans les cholangiocytes, on peut craindre que le tamoxifène induise une expression ectopique de CrekR dans les hépatocytes, perturbant ainsi l'interprétation des données de traçage. Un renouvellement d'hépatocytes à partir de cellules précurseurs dépourvues d'albumine semble cependant bien détectable. Toutefois, le taux de renouvellement calculé de $0,076 \%$ tous les 4 jours [22] semble incompatible avec le modèle proposé par Uemoto qui prévoit un renouvellement complet des hépatocytes en un an. Enfin, un traçage de lignée des hépatocytes par marquage des cellules au moyen d'un système viral induisant l'expression de la Cre recombinase spécifiquement dans les hépatocytes, ne révèle pas de renouvellement significatif de ces cellules à partir de cholangiocytes [23]. Il est donc probable que le taux de renouvellement cellulaire soit faible dans le foie adulte, et qu'il résulte principalement d'un phénomène de prolifération intrinsèque à l'hépatocyte mature. Une différenciation à partir de cellules précurseurs n'est pas à exclure, mais reste sans doute quantitativement peu importante en conditions homéostatiques.
En conditions pathologiques, le tableau est différent. En réponse à une hépatectomie partielle, le foie régénère principalement par prolifération cellulaire, les hépatocytes donnant naissance à de nouveaux hépatocytes. Toutefois, si une atteinte hépatique s'accompagne d'une inhibition de la prolifération des hépatocytes, la régénération implique l'induction de cellules souches. Cette induction se manifeste chez les rongeurs par la présence de cellules ovales, dont le nom provient de l'aspect ovale du noyau. Ces cellules colonisent les régions périportales du foie. Elles présentent un profil d'expression génique mixte, à la fois cholangiocytaire et hépatocytaire, et sont considérées comme des intermédiaires entre les cellules souches et les cellules épithéliales différenciées (transit amplifying cells). Chez l'homme, une affection aiguë du foie entraîne une réaction ductulaire, homologue à l'induction de cellules ovales chez les rongeurs [24]. L'origine des cellules ovales fait l'objet d'un débat depuis longtemps [25]. Elles dérivent probablement de cellules souches situées dans les passages de Hering, et au moins une partie des cellules délimitant ces passages est issue de la plaque ductale [15]. Au total, dans un foie adulte en condition pathologique, les hépatocytes dérivent d'hépatocytes préexistants ou de cellules progénitrices, selon la nature de l'atteinte hépatique.

Qu'en est-il enfin des cholangiocytes en pathologie? Ici également, la controverse règne. Chez des rongeurs dont les voies biliaires ont été lésées, la transplanta- 


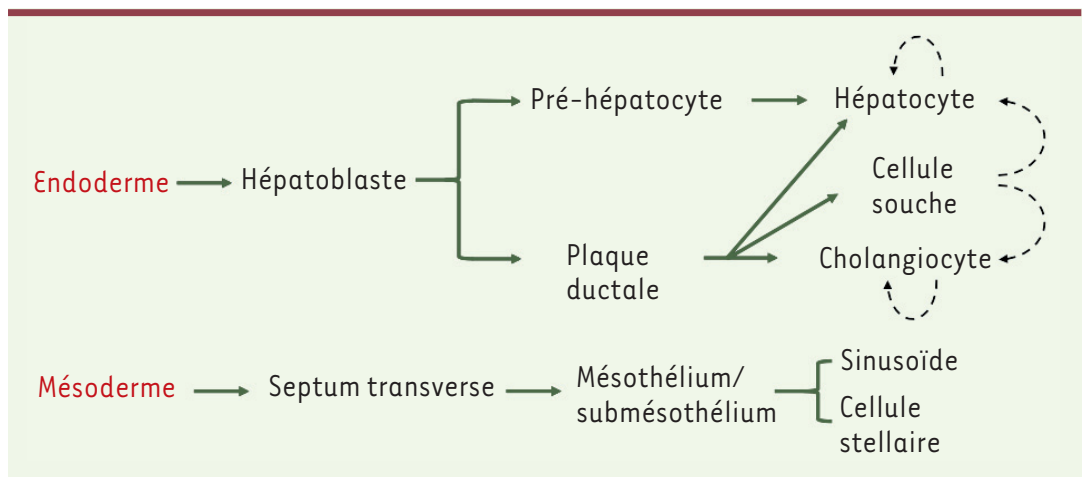

Figure 4. Développement des lignages hépatiques. L'endoderme donne naissance aux hépatoblastes, ces derniers étant la source de cholangiocytes, d'hépatocytes et de cellules souches adultes. En cas d'atteinte du foie, les hépatocytes et cholangiocytes se régénèrent par prolifération ou par différenciation à partir de cellules souches. Le mésoderme donne naissance aux cellules stellaires et sinusoïdales, par l'intermédiaire du septum transverse.

tion d'hépatocytes identifiables génétiquement aboutit à la constitution de voies biliaires bordées de cholangiocytes porteurs de la marque génétique, suggèrant que les hépatocytes peuvent subir une transdifférenciation vers le lignage cholangiocytaire [24]. Cette interprétation n'est cependant pas acceptée par tous. Les chercheurs qui ont réalisé le traçage d'hépatocytes marqués au moyen d'une infection virale ne détectent, après lésion des voies biliaires, aucun cholangiocyte dérivé d'hépatocytes [23]. II y a toutefois des raisons de penser que les différentes études ne se contredisent pas. II est en effet probable que la nature de la lésion hépatique détermine la nature de la réponse cellulaire. La plasticité des cellules hépatiques détectée chez l'embryon et chez l'adulte, en condition normale ou pathologique, commence seulement à révéler ses premiers secrets. $\diamond$

\section{SUMMARY}

Lineage fate decisions in normal and regenerating liver

The origin of hepatocytes and cholangiocytes in embryonic and adult liver, as well as the source of stellate and sinusoidal cells, have received much attention recently, due to the availabilty of biological tools enabling to trace the fate of cell lineages. A model is now proposed which defines the differentiation potential of hepatoblasts, ductal plate cells and adult progenitor cells, in normal conditions and in regenerating liver. The mesodermal origin of stellate and sinusoidal cells is also established. $\diamond$

\section{LIENS D'INTÉRÊT}

L'auteur déclare n'avoir aucun lien d'intérêt concernant les données publiées dans cet article.

\section{RÉFÉRENCES}

1. Lemaigre FP. Mechanisms of liver development : concepts for understanding liver disorders and design of novel therapies. Gastroenterology $2009 ; 137: 62-79$.

2. Si-Tayeb K, Lemaigre FP, Duncan SA. Organogenesis and development of the liver. Dev Cell 2010 ; $18: 175-89$.

3. Germain L, Blouin MJ, Marceau N. Biliary epithelial and hepatocytic cell lineage relationships in embryonic rat liver as determined by the differential expression of cytokeratins, alphafetoprotein, albumin, and cell surface-exposed components. Cancer Res $1988 ; 48: 4909-18$.

4. Roskams T, Desmet V. Embryology of extra- and intrahepatic bile ducts, the ductal plate. Anat Rec (Hoboken) $2008 ; 291: 628-35$

5. Clotman F, Lannoy VJ, Reber M, et al. The onecut transcription factor HNF6 is required for normal development of the biliary tract. Development $2002 ; 129$ : 1819-28.

6. Clotman F, Jacquemin P, Plumb-Rudewiez N, et al. Control of liver cell fate decision by a gradient of TGF beta signaling modulated by Onecut transcription factors. Genes Dev $2005 ; 19: 1849-54$.
7. Hunter MP, Wilson CM, Jiang X, et al. The homeobox gene Hhex is essential for proper hepatoblast differentiation and bile duct morphogenesis. Dev Biol $2007 ; 308: 355-67$.

8. Lorent K, Yeo SY, Oda T, et al. Inhibition of Jagged-mediated Notch signaling disrupts zebrafish biliary development and generates multi-organ defects compatible with an Alagille syndrome phenocopy. Development $2004 ; 131$ : 5753-66.

9. Clotman F, Libbrecht L, Killingsworth MC, et al. Lack of cilia and differentiation defects in the liver of human foetuses with the Meckel syndrome. Liver Int $2008 ; 28: 377-84$.

10. Antoniou A, Raynaud P, Cordi S, et al. Intrahepatic bile ducts develop according to a new mode of tubulogenesis regulated by the transcription factor SOX9. Gastroenterology $2009 ; 136: 2325-33$.

11. Van Eyken P, Sciot R, Callea F, et al. The development of the intrahepatic bile ducts in man : a keratin-immunohistochemical study. Hepatology 1988 ; 8 : 1586-95.

12. Raynaud P, Carpentier R, Antoniou A, Lemaigre FP. Biliary differentiation and bile duct morphogenesis in development and disease. Int J Biochem Cell Biol $2011 ; 43: 245-56$.

13. Crawford AR, Lin XZ, Crawford JM. The normal adult human liver biopsy : a quantitative reference standard. Hepatology $1998 ; 28$ : 323-31.

14. Terada T, Nakanuma Y. Detection of apoptosis and expression of apoptosisrelated proteins during human intrahepatic bile duct development. Am J Pathol $1995 ; 146: 67-74$.

15. Carpentier R, Suner RE, Van Hul N, et al. Embryonic ductal plate cells give rise to cholangiocytes, periportal hepatocytes, and adult liver progenitor cells. Gastroenterology $2011 ; 141$ : 1432-8.

16. Kopp JL, Dubois CL, Schaffer AE, et al. Sox $9^{+}$ductal cells are multipotent progenitors throughout development but do not produce new endocrine cells in the normal or injured adult pancreas. Development $2011 ; 138: 653-65$.

17. Asahina K, Tsai SY, Li P, et al. Mesenchymal origin of hepatic stellate cells, submesothelial cells, and perivascular mesenchymal cells during mouse liver development. Hepatology 2009 ; 49 : 998-1011.

18. Asahina K, Zhou B, Pu WT, Tsukamoto H. Septum transversum-derived mesothelium gives rise to hepatic stellate cells and perivascular mesenchymal cells in developing mouse liver. Hepatology $2011 ; 53$ : 983-95.

19. Cassiman D, Barlow A, Vander Borght S, et al. Hepatic stellate cells do not derive from the neural crest.J Hepatol 2006 ; $44: 1098-104$.

20. Perez-Pomares JM, Carmona R, Gonzalez-Iriarte M, et al. Contribution of mesothelium-derived cells to liver sinusoids in avian embryos. Dev Dyn $2004 ; 229: 465-74$.

21. Furuyama K, Kawaguchi $\mathrm{Y}$, Akiyama $\mathrm{H}$, et al. Continuous cell supply from a Sox9-expressing progenitor zone in adult liver, exocrine pancreas and intestine. Nat Genet $2011 ; 43: 34-41$.

22. Iverson SV, Comstock KM, Kundert JA, Schmidt $\varepsilon$. Contributions of new hepatocyte lineages to liver growth, maintenance, and regeneration in mice. Hepatology $2011 ; 54: 655-63$.

23. Malato $\mathrm{Y}$, Naqvi S, Schurmann $\mathrm{N}$, et al. Fate tracing of mature hepatocytes in mouse liver homeostasis and regeneration. J Clin Invest 2011 ; 121 : 4850-60.

24. Michalopoulos GK. Liver regeneration : alternative epithelial pathways. Int J Biochem Cell Biol $2011 ; 43: 173-9$.

25. Laperche Y. Origine des cellules ovales hépatiques. Med Sci (Paris) 2007 ; $23: 480-2$.

\section{TIRÉS À PART}

F. Lemaigre 